\title{
Synthesis of 3-(Trimethoxysilyl) Propyl Methacrylate Functionalized Graphene Oxide Based Mixed Matrix Membrane and Its Application for $\mathrm{O}_{2} / \mathrm{N}_{2}$ Separation
}

\author{
Shahnila Shah ${ }^{* 1}$, Huma Shaikh ${ }^{1}$, Sarah Hafeez ${ }^{2}$ and Muhammad Imran Malik ${ }^{3}$ \\ ${ }^{1}$ National Center of Excellence in Analytical Chemistry, University of Sindh, Jamshoro, Pakistan. \\ ${ }^{2}$ School of Chemical and Materials Engineering (SCME), National University of Sciences \& Technology, \\ Islamabad, Pakistan. \\ ${ }^{3}$ H.E.J. Research Institute of Chemistry, International Centre for Chemical and Biological Sciences (ICCBS), \\ University of Karachi, Karachi, Pakistan. \\ *Corresponding Author Email: shahnila_s@yahoo.com \\ Received 14 February 2020, Revised 20 June 2020, Accepted 22 June 2020
}

\begin{abstract}
The incorporation of functionalized graphene oxide (GO) in mixed matrix membrane (MMM) is expected to greatly increase the permeability and selectivity for $\mathrm{O}_{2} / \mathrm{N}_{2}$ separation. In the present study, GO functionalized with 3- (Trimethoxysilyl) propyl methacrylate (TMOPMA) was used as inorganic filler and incorporated in to a PVC/pAMPS based MMM to increase the separation efficiency. Membranes of different compositions were synthesized and the best morphology was achieved with $0.5 \mathrm{~g}$ of PVC, $1.0 \mathrm{~g}$ of pAMPS and $0.015 \mathrm{~g}$ of filler. The synthesized membrane and inorganic filler were characterized using SEM, EDS, FTIR, Raman and XRD spectroscopy. Moreover, the gas permeation studies were performed to check the separation factor of synthesized membrane for $\mathrm{O}_{2} / \mathrm{N}_{2}$. The maximum permeability achieved for $\mathrm{O}_{2}$ and $\mathrm{N}_{2}$ was 4097 and 3373 barrers, respectively at 5 bar pressure. For the selectivity, a gradual increasing trend was observed with the increase in permeability. The maximum selectivity achieved was 1.215 at 5 bar pressure. The results revealed an increasing trend in selectivity with the increase in permeability of gases across the membrane with the increase in feed gas pressure.
\end{abstract}

Keywords: $\mathrm{O}_{2} / \mathrm{N}_{2}$ separation, Silylation, Graphene oxide, Mixed matrix membranes.

\section{Introduction}

In recent years air separation became a widely used commercial process due to the great applicability of $\mathrm{O}_{2}$ and $\mathrm{N}_{2}$ gases in different industrial processes. Conventionally, the gas separation was achieved using highly power consumption systems such as; cryogenic treatment, solute/solvent adsorption etc. [1]. However, these days more efficient and cost effective membrane-based separations are evolved as widely accepted techniques for the gas separation. During past few decades, in membranes-based refinement of gases, where the range of membrane materials is available, the development of polymeric membranes for selective transportation of one gas from a mixture has fascinated considerable attention of scientists. The interest in using polymeric membranes for selective gas permeation is based on their number of advantages such as; processing feasibility, cost effectiveness, structural regularity and more important a trade-off could be achieved between selectivity and gas permeability using polymeric membranes $[2,3]$. The further improvement in the properties has been resulted due to the substantial research in gas separation properties of polymeric membranes [4]. In membrane based techniques, separation is achieved due to the difference of oxygen and nitrogen diffusion rates through the 
membrane which allow us to acquire high $\mathrm{O}_{2}$ permeability and $\mathrm{O}_{2} / \mathrm{N}_{2}$ selectivity. As shown in Fig. 1, $\mathrm{O}_{2}$ rich stream act as permeate while $\mathrm{N}_{2}$ enriched stream is rentetate and can be recovered at high-pressure side of module. Since, the difference between the kinetic diameters of $\mathrm{O}_{2}$ and $\mathrm{N}_{2}$ is very low (3.46 $\AA$ and $3.64 \AA$, respectively), it is very difficult to separate them out as compare to other gases. Numerous attempts have been made to improve the permselectivity of both gases [5].

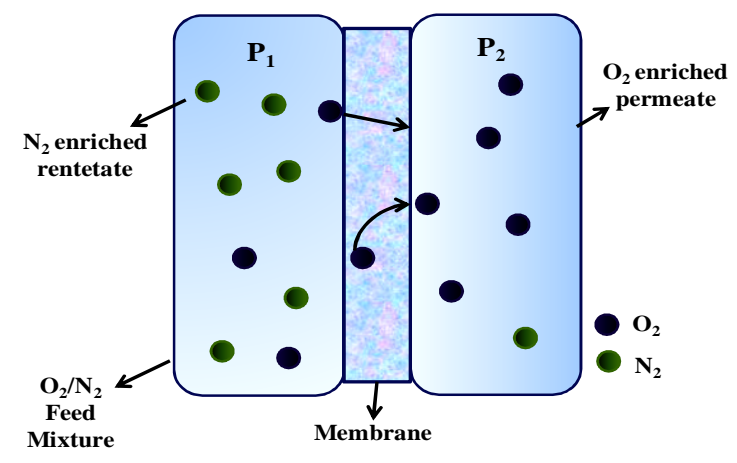

Figure 1. Separation of $\mathrm{O}_{2} / \mathrm{N}_{2}$ through polymeric membrane

A whole new era of progression of highly efficient gas separation membranes has opened up with the advent of GO based membranes. Because of its unique properties, GO has fascinated significant consideration lately compared with conventional nanofillers, it has amazing properties while the high surface area is an advantage for great interface between the nanofillers and membrane materials, it also provides superior mechanical strength [6,7]. Due to the presence of different functional groups (i.e. epoxide, carboxyl, hydroxyl) on the surface of single atom thick GO monolayer; which are appropriate anchoring points for further required functionalization, GO based membranes exhibits great potential application in separation applications [8,9].

The gases separation using GO based membranes is mainly followed by sieving mechanism arises due to packing and structural defects in the inter-layer channels [10]. These structural voids are suitable for molecular separation according to the differences in size and facilitate the speedy transportation of certain molecules through the inter layers spacing [11, 12]. Consequently, by controlling the density and defects of GO sheets their separation performance can be further improved [7].
Assuming that the laminate structure of GO based membranes is composed of highly compact nanosheets which on interaction with nanofillers such as nanoparticles, nanowires, MOFs etc, can effectually expand the gaps, thus offers the speedy transportation of gas molecules through the channels $[13,14]$. To ensure the good separation factor of the GO-based composite membranes the interfacial contact between the intercalated filler and membrane should be in such way that it guarantee that selectivity of membrane would not be sacrificed by the generation of nonselective voids in membrane [15].

Silane based coupling agents have attracted great interest recently as a compatibilizer for enhancing the interfacial adherence between GO sheets and polymer [6]. Moreover, the solution-diffusion model is based on the fact that, the permeability of a gas is directly related to its solubility in membrane. To achieve the higher permeability of oxygen various materials have been suggested, among them the contribution of $\mathrm{Si}-\mathrm{O}-$ bonds have gained the considerable attention to achieve the higher solubility of oxygen $[16,17]$.

The present study illustrates the use of 3(Trimethoxysilyl) propyl methacrylate (TMOPMA) to modify the surface of GO to enhance the $\mathrm{O}_{2} / \mathrm{N}_{2}$ separation performance of membrane. The silane modified GO (S-GO) was embedded into PVC/pAMPS based mixed matrix membrane (MMM) as nanofiller. The choice of MMM for this study was based on the fact that composite membranes, in which solid adsorbents or fillers are added to enhance membrane performance for gas separation are getting boost day by day. Moreover, the selection of membrane's precursors was based on coupling of their good separation performance with superior chemical and physical properties and high mechanical strength of membranes. The morphological studies of fabricated membranes were performed by SEM, elemental compositions were confirmed by performing EDS, whereas qualitative analysis of membranes were performed by FTIR, Raman and XRD spectroscopy. The gas permeation studies were performed to check the applicability of synthesized membranes for $\mathrm{O}_{2} / \mathrm{N}_{2}$ separation. 
Materials and Methods Materials

Graphite flakes, hydrochloric acid, (Contry). Sulphuric acid and potassium permanganate was purchased from Alfa Aesar. 3(Trimethoxysilyl) propyl methacrylate, N, N'Dimethylformamide, 2-acrylamido-2-methyl- 1propanesulfonic acid (AMPS) and sodium nitrate were bought from Sigma-Aldrich, USA. Sodium sulfite, hydrogen Peroxide, ammnium Iron (II) sulfate and ammonium per sulfate were procured from Duksan (Pure chemicals), Korea. Polyvinyl chloride (PVC) and ethanol were bought from Fluka Chemika, Switzerland.

\section{Synthesis of graphene oxide}

Compact sheets of GO were synthesized by following modified Hummers' method [18]. For that in $35 \mathrm{~mL}$ of $98 \%$ conc. $\mathrm{H}_{2} \mathrm{SO}_{4}, 1 \mathrm{~g}$ of $\mathrm{NaNO}_{3}$ and $2 \mathrm{~g}$ of graphite were added and stirred for mixing. The temperature of mixture was set to $0^{\circ}$ in an ice-bath. Continuing the vigorous stirring, $6 \mathrm{~g}$ of $\mathrm{KMnO}_{4}$ was mixed to the suspension. The temperature of the suspension was controlled to exceed from $2^{\circ}$, which was then brought to $35 \pm 3^{\circ}$ by removing the ice-bath. $400 \mathrm{~mL}$ of DI water was then added slowly to the solution and stirred for half an hour. Finally the suspension was treated with $5 \mathrm{~mL}$ of $30 \% \mathrm{H}_{2} \mathrm{O}_{2}$ that result the change in the color indicating the reaction completion. The solution was then centrifuged and washed with 5\% $\mathrm{HCl}$ and DI water to achieve the neutral $\mathrm{pH}$.

\section{Silylation of graphene oxide}

After the synthesis of GO, silylation of GO was performed (Fig. 2.) in 1:1 solution of water and methanol, for that $100 \mathrm{mg}$ of GO was dispersed in $2.5 \mathrm{~mL}$ of water via $4 \mathrm{~h}$ sonication at room temperature. 50\% solution of TMOPMA was prepared in $2.5 \mathrm{~mL}$ methanol and then added to the dispersed GO. Resulting reaction mixture was again sonicated for $4 \mathrm{~h}$ and kept under room temperature for complete modification. After $48 \mathrm{~h}$ the solution was washed out with methanol in centrifuged at $7200 \mathrm{rpm}$ at $25{ }^{\circ} \mathrm{C}$ for $30 \mathrm{~min}$ per cycle. After several washes the sample was dried in a petri dish.

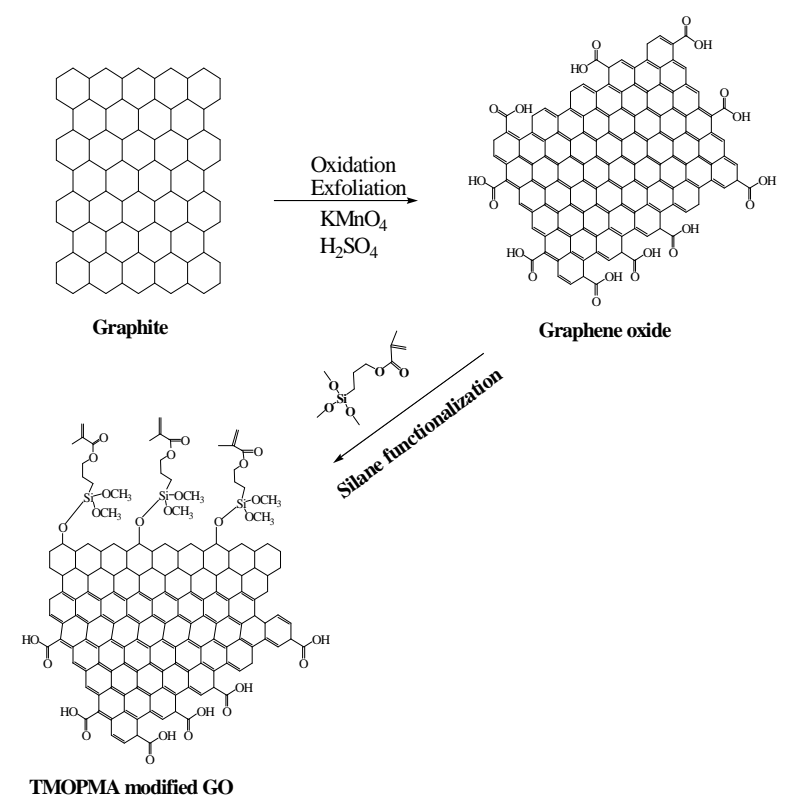

Figure 2. Schematic representation of synthesis of S-GO

\section{Fabrication of Mited matrix membrane}

For membrane preparation, Poly (2acrylamido-2-methyl- 1-propanesulfonic acid) (pAMPS) was prepared first by following a reported method [19]. The polymerization was carried out in $20 \%$ aqueous solution of monomer (AMPS) at $25{ }^{\circ} \mathrm{C}$ for $15 \mathrm{~h}$ through free radical polymerization by applying the redox-initiator system containing sodium sulfite, APS and ammonium iron (II) sulfate. After $15 \mathrm{~h}$ of polymerization, the obtained pAMPS was air dried and stored in a cool place.

Membranes were then fabricated using solution casting procedure [20]. For that, different amounts of PVC and pAMPS were dissolved in 5 $\mathrm{mL}$ of DMF in separate beakers and placed on magnetic mixer for $1 \mathrm{~h}$. After complete mixing both the solutions were mixed and stirred using magnetic stirrer for $24 \mathrm{~h}$ at room temperature. Various solutions were prepared in different beakers for pure and nanofiller i.e., S-GO embedded polymeric membranes. The blended mixture was kept unmoved for $2 \mathrm{~h}$ to remove the bubbles. The resulting solution was cast on a petri dish, placed in oven at $80{ }^{\circ} \mathrm{C}$ for $48 \mathrm{~h}$ to evaporate the solvent (Fig. 3). The resultant membrane was separated out from glass surface by dipping in water. 


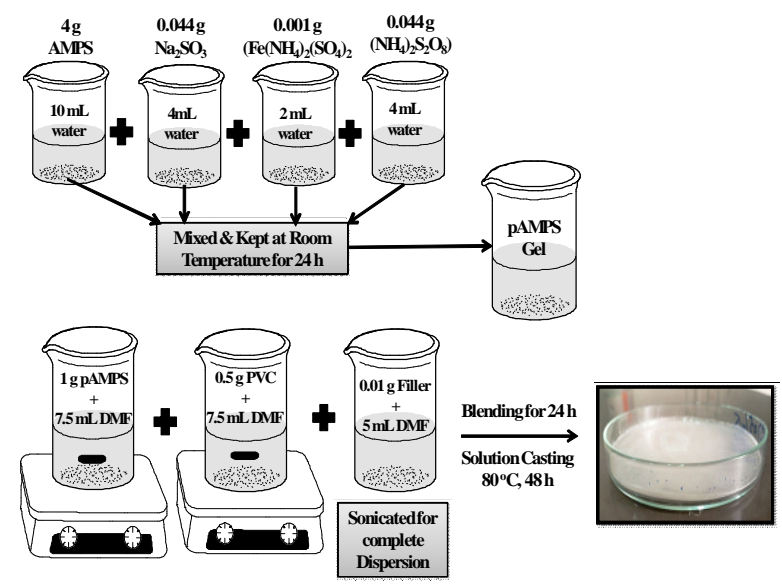

Figure 3. Flow chart for fabrication of S-GO embedded MMM

\section{Characterization}

The surface morphology and elemental analysis of synthesized membrane was studied by taking the surface images using SEM (JEOL JSM6380LV) attached with EDS. Qualitative structural analysis of synthesized membrane and S-GO was performed by using FTIR spectrophotometer (FTS-65, Biorad) with resolution of $1 \mathrm{~cm}^{-1}$ and wave numbers from 450 to $4000 \mathrm{~cm}^{-1}$. The small circular portion of filler embedded membrane was placed in a sample holder and mounted on instrument to record the spectra. Raman spectroscopy was performed using Thermo Scientific DXR Raman microscope with $780 \mathrm{~nm}$ filter laser source and a CCD detector. XRD patterns were investigated to analyze the structural characteristics of nanofiller. For this purpose STOE Germany Theta-Theta diffractometer (Germany, software, WinXPoe X'Pert High Score) with $\mathrm{Cu} \mathrm{K \alpha}$ monochromatic radiations (wavelength $=1.54 \mathrm{~A}^{\circ}$ ) was used.

Applicability of the synthesized membranes was checked for pure gas permeability and selectivity of polymeric membrane for separation of $\mathrm{O}_{2}$ and $\mathrm{N}_{2}$ by performing gas separation experiments under isothermal conditions at $35{ }^{\circ} \mathrm{C}$ using permeation testing setup embellished by Hafeez S. et al., [21] elaborated in Fig. 4. Permeability of a gas can be measured by using equation 1 and is generally expressed in barrer $\left(10^{-10}\left[\mathrm{~cm}^{3}(\mathrm{STP}) \mathrm{cm} /\left(\mathrm{cm}^{2} \mathrm{~s} \mathrm{~cm} \mathrm{Hg}\right)\right]\right)$. The ideal separation factor was calculated by taking the ratio of permeabilities for gas $\mathrm{A}$ and $\mathrm{B}$ (equation 2):

$$
\mathrm{P}=\frac{\mathrm{QL}}{\Delta \mathrm{PA}}
$$

Where $\mathrm{Q}$ flow rate $(\mathrm{mL} / \mathrm{min}), \mathrm{A}$ is the effective membrane area in $\left(\mathrm{m}^{2}\right), \mathrm{L}$ is thickness $(\mathrm{m})$ and $\Delta \mathrm{P}$ is the pressure difference (bar) between high pressure (HP) and low pressure (LP).

$\mathrm{a}_{\frac{\mathrm{A}}{\mathrm{B}}}=\frac{\mathrm{P}_{\mathrm{A}}}{\mathrm{P}_{\mathrm{B}}}$

Where $\mathrm{P}_{\mathrm{A}}$ is the permeability of oxygen and $\mathrm{P}_{\mathrm{B}}$ represents the nitrogen's permeability.

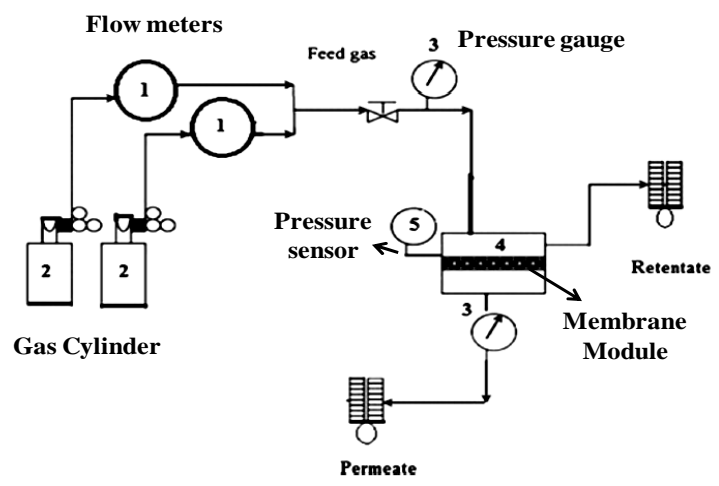

Figure 4. Schematic representation of gas permeation setup

\section{Results and Discussion}

Optimization of amounts of polymers and nanofiller

Homogeneity and miscibility of the blend components play important role in determining the applicability of a polymer blend [22, 23]. In order to get the membranes with the best morphology, different compositions of membranes were prepared. Table 1 shows the different amounts of PVC and pAMPS checked for the synthesis of MMMs and the best composition was obtained at $0.5 \mathrm{~g}$ of PVC and $1 \mathrm{~g}$ of pAMPS which resulted the membranes having thickness of $\sim 40-50 \mu \mathrm{m}$. As the amount of PVC was increased the thickness of membrane was also increased that causes the decrease in permeability. Similarly, the amounts of nanofillers were also optimized by preparing several membranes having different amounts of nanofillers and the best composition was obtained with $0.015 \mathrm{~g}$ (Table 2). As the amount lower than this was causing irregular embedding of nanofiller all over the membrane and the amount higher than this value was causing the overloading and thus rupturing of membranes. 
Table 1. Optimization of PVA/pAMPS amounts.

$\begin{array}{lllllllllll}\boldsymbol{P V C}(\mathrm{g}) & 1.0 & \mathbf{0 . 5} & 1.0 & 0.5 & 0.25 & 0.25 & 0.5 & 0.125 & 0.063 & 0.03\end{array}$

$\begin{array}{ccccccccccc}\underset{(g)}{p A M P S} & 1.0 & \mathbf{1 . 0} & 0.5 & 0.5 & 0.5 & 0.25 & 0.25 & 0.25 & 0.125 & 0.063\end{array}$

Table 2. Optimization of nanofillers amount.

\begin{tabular}{lccccccc}
\hline $\boldsymbol{P V C}(\boldsymbol{g})$ & 0.5 & 0.5 & 0.5 & 0.5 & 0.5 & 0.5 & 0.5 \\
\hline $\boldsymbol{p A M P S}$ & 1.0 & 1.0 & 1.0 & 1.0 & 1.0 & 1.0 & 1.0 \\
\begin{tabular}{l}
$(\boldsymbol{g})$ \\
\hline $\begin{array}{l}\text { Nanofiller } \\
(\boldsymbol{g})\end{array}$
\end{tabular} & 0.5 & 0.25 & 0.125 & 0.063 & 0.03 & 0.015 & 0.007 \\
\hline
\end{tabular}

The surface morphology of GO and membrane embedded with S-GO can be observed in (Fig. 5a-b). Fig.5a shows the 2D morphology of unstacked nanosheet with wrinkled and folded textures in graphene oxide sheets. Whereas, Fig. $5 \mathrm{~b}$ corresponds to the membrane fabricated with S-GO. SEM image of dense membrane shows the homogenous allocation and effectual adhesion between nanofiller and polymer matrix. However, generation of voids was also observed in surface demonstrating the increase in free volume in the polymeric channels. This is most likely due to the disturbance in packing of polymeric chains due to its interaction with inorganic nanofiller which may enhance the gaps generation [24].
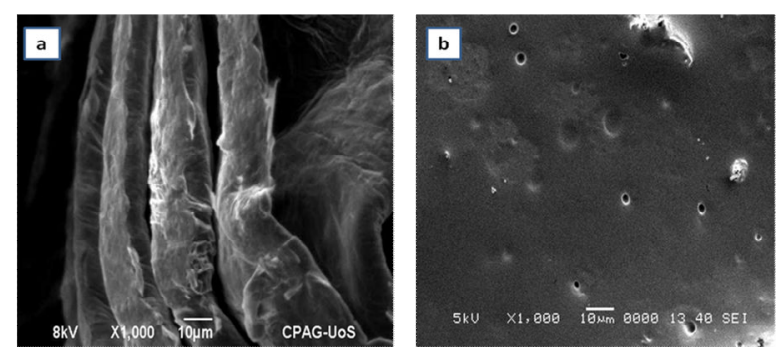

Figure 5. SEM images a) GO, b) S-GO embedded MMM

The elemental composition of S-GO and membrane fabricated with S-GO was examined by performing EDS analysis. Fig. 6a shows the EDS spectra of S-GO which along with the peaks of C and $\mathrm{O}$ having the $\mathrm{K} \alpha$ values of 0.277 and 0.525 , respectively, showing the characteristic peak of $\mathrm{Si}$ with the $\mathrm{K} \alpha$ value of 1.74 ; which gives the initial confirmation of modification of GO with TMOPMA. Fig. 6b corresponds to the EDS spectra of membrane embedded with S-GO, which along with the distinctive peaks of $\mathrm{N}, \mathrm{S}$ and $\mathrm{Cl}$ from membrane materials having the $\mathrm{k} \alpha$ values of $0.392,2.307$ and 2.621, respectively, showing all the other characteristic peaks of GO and Si. $\mathrm{K} \alpha$ values of each obtained peak were compared with the energy table and exact matching of each value was found. Thus, confirming the successful fabrication of membranes with nanofiller.
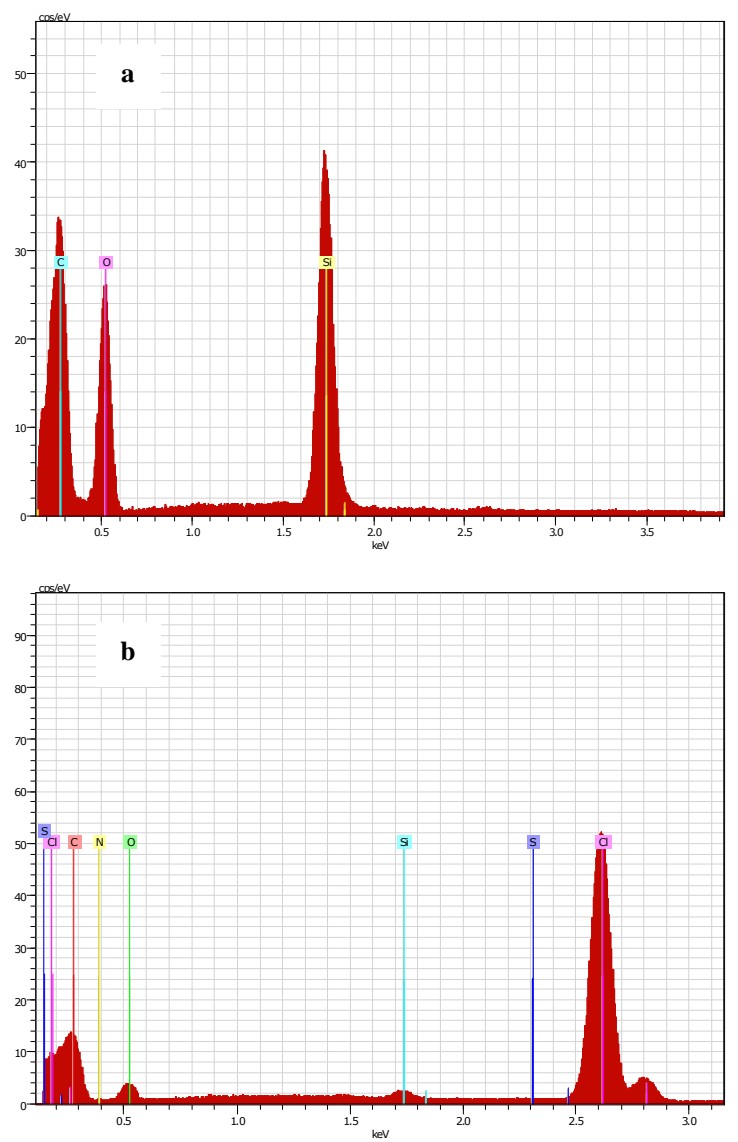

Figure 6. EDS analysis of a) S-GO, b) S-GO embedded MMM

Fig. 7a-b correspond to the FTIR spectra of S-GO and membrane blended with S-GO, respectively. In spectra (a) the main peaks at 3383 $\mathrm{cm}^{-1}$ and $2950 \mathrm{~cm}^{-1}$ signify the stretching of $\mathrm{OH}$ and C-H alkyl groups, respectively. A sharp peak appearing at $1719 \mathrm{~cm}^{-1}$ can be assigned as characteristic band for $\mathrm{C}=\mathrm{O}$ group. The characteristic peaks from silane could be observed in the spectra at different wavenumbers; the peak 
at $850 \mathrm{~cm}^{-1}$ signifies the presence of $\mathrm{Si}-\mathrm{O}-\mathrm{CH}_{2}$ group and two asymmetric peaks at $1169 \mathrm{~cm}^{-1}$ and $1122 \mathrm{~cm}^{-1}$ represent the $\mathrm{Si}-\mathrm{O}$ bending and $\mathrm{O}=\mathrm{C}-\mathrm{O}-$ $\mathrm{C}$ aliphatic stretch [25]. In the spectrum of membrane (Fig 7b) again the main peaks at 3461 $\mathrm{cm}^{-1}$ and 3423 come from modified graphene oxide and are typically of alcoholic and carboxylic hydroxyl groups, respectively. An intense peak in the region of $1217 \mathrm{~cm}^{-1}$ is possibly due to the presence of silane bonded with three oxygen atom as in TMOPMA. A strong stretching of $\mathrm{C}-\mathrm{Cl}$ could be seen at $772 \mathrm{~cm}^{-1}$ comes from PVC. Whereas the small peak appearing at $1441 \mathrm{~cm}^{-1}$ is attributed to $\mathrm{S}=\mathrm{O}$ groups of pAMPS. This confirms the effective embedding of nanofiller into the membrane.
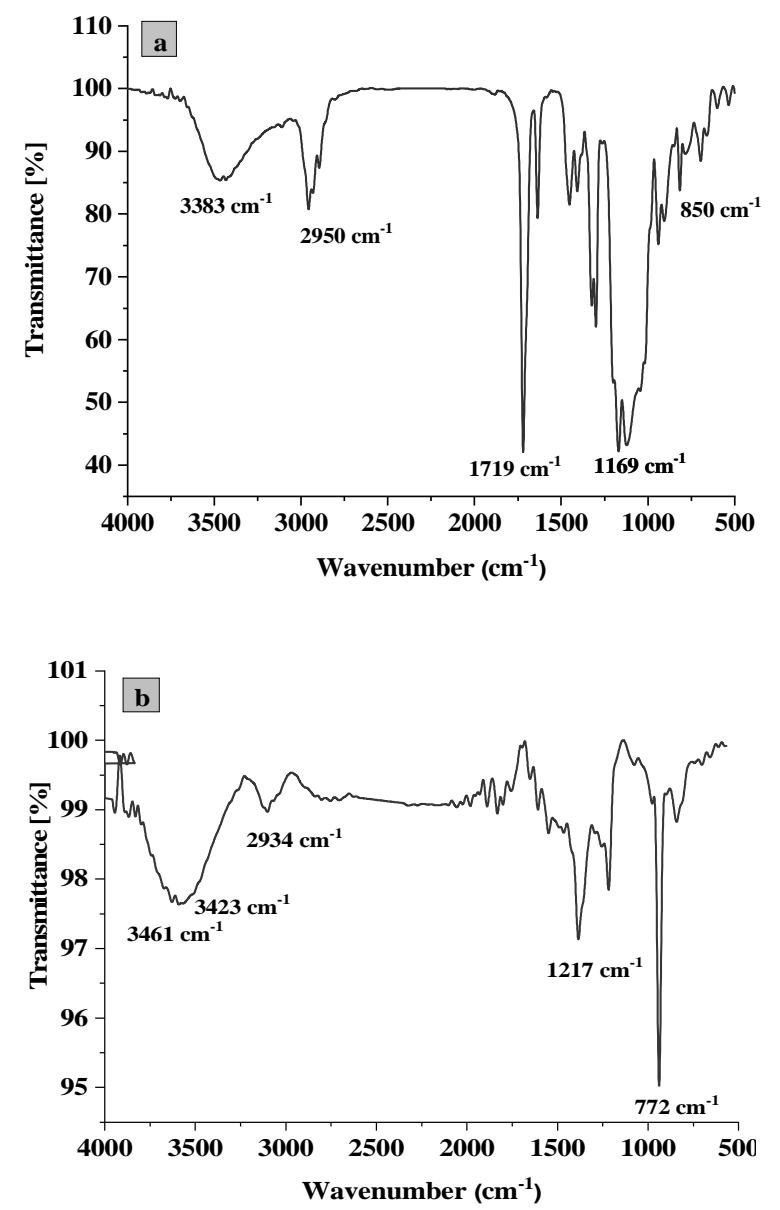

Figure 7. FTIR spectra of a) S-GO b) S-GO embedded MMM

Raman spectroscopy is a greatly accepted technique for the characterization of conjugated and double bonded carbon compounds which gives intense raman peaks [26]. The noteworthy structural changes that occur during the synthesis and modification of GO are reflected in Fig. 8. In $\mathrm{GO}$ (a), the broadening and shifting of $\mathrm{G}$ band was observed at $1600 \mathrm{~cm}^{-1}$ and a D band appears at $1330 \mathrm{~cm}^{-1}$ because of the reduction in size of inplane $\mathrm{sp}^{2}$ domains of GO as compared to that of pristine graphite. The raman spectrum of S-GO also exhibits both $\mathrm{G}$ and $\mathrm{D}$ bands at 1590 and 1310 $\mathrm{cm}^{-1}$, respectively but with an increased intensity ratio. As this ratio is inversely related to the crystallite size, this alteration directed a decrease in the average size of the $\mathrm{sp}^{2}$ domains upon intercalation of nanofiller in exfoliated sheets of GO due to the breakdown of the k-selection rule [27].
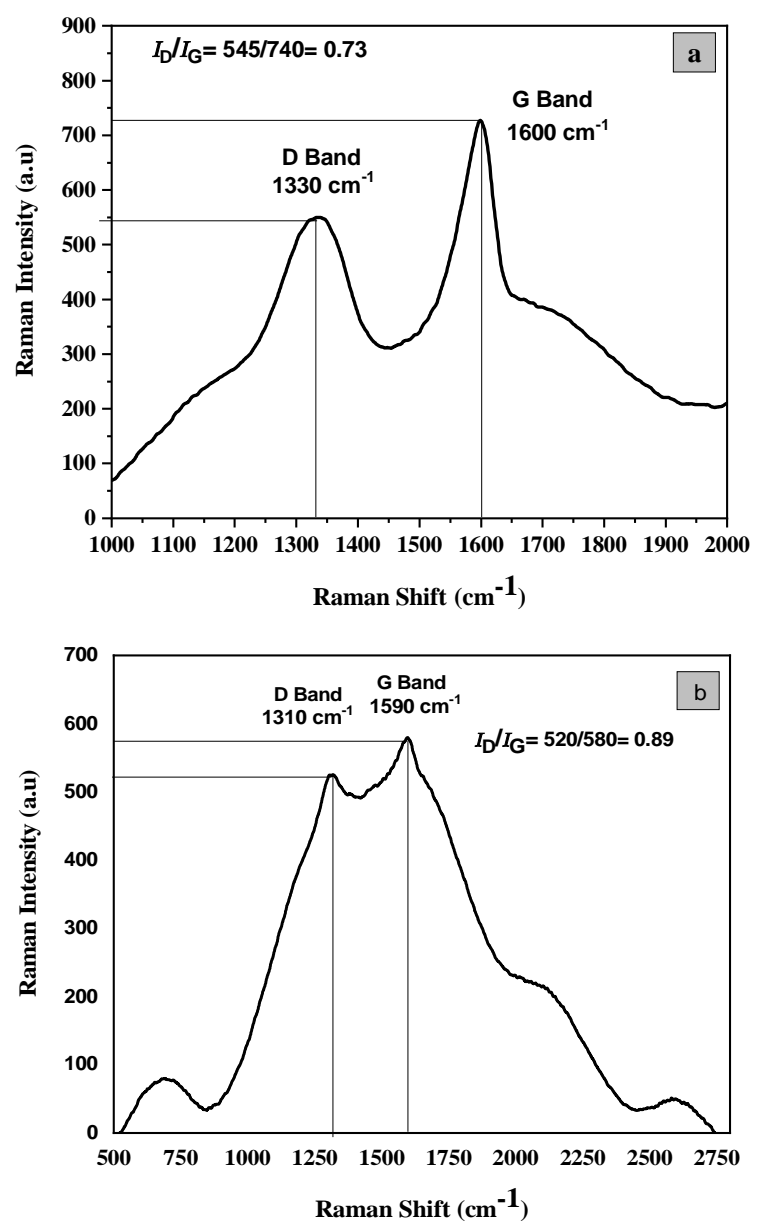

Figure 8. Raman spectra a) GO, b) S-GO

XRD analysis was used to get information regarding the stacking of GO sheets and the 
estimation of degree of exfoliation in GO sheets. XRD patterns of GO and S-GO are represented in Fig. 9. The reported XRD pattern of graphite shows a strong diffraction peak at $2 \theta=26.52^{\circ}$ (002), representing an interlayer spacing of 0.34 $\mathrm{nm}$ [28]. This peak was disappeared in GO (Fig. 9a) and a new peak was observed at $2 \theta=11.8^{\circ}$ representing an interlayer spacing of $0.75 \mathrm{~nm}$ as evaluated by the Bragg's equation [29]. This increase in interlayer spacing is due to the presence of different functional groups in GO [30]. Moreover, after modification of GO with TMOPMA there is a shift in the $2 \theta$ to $10.5^{\circ}$ with an interlayer spacing of $0.84 \mathrm{~nm}$ this is due to the fact that after insetting of silane functionalization in between the GO sheets could greatly enhance the interlayer spacing [31]. However the intensity of peak is much lower as compared to that of GO this reflects the loss of crystallinity of GO after its modification [32].
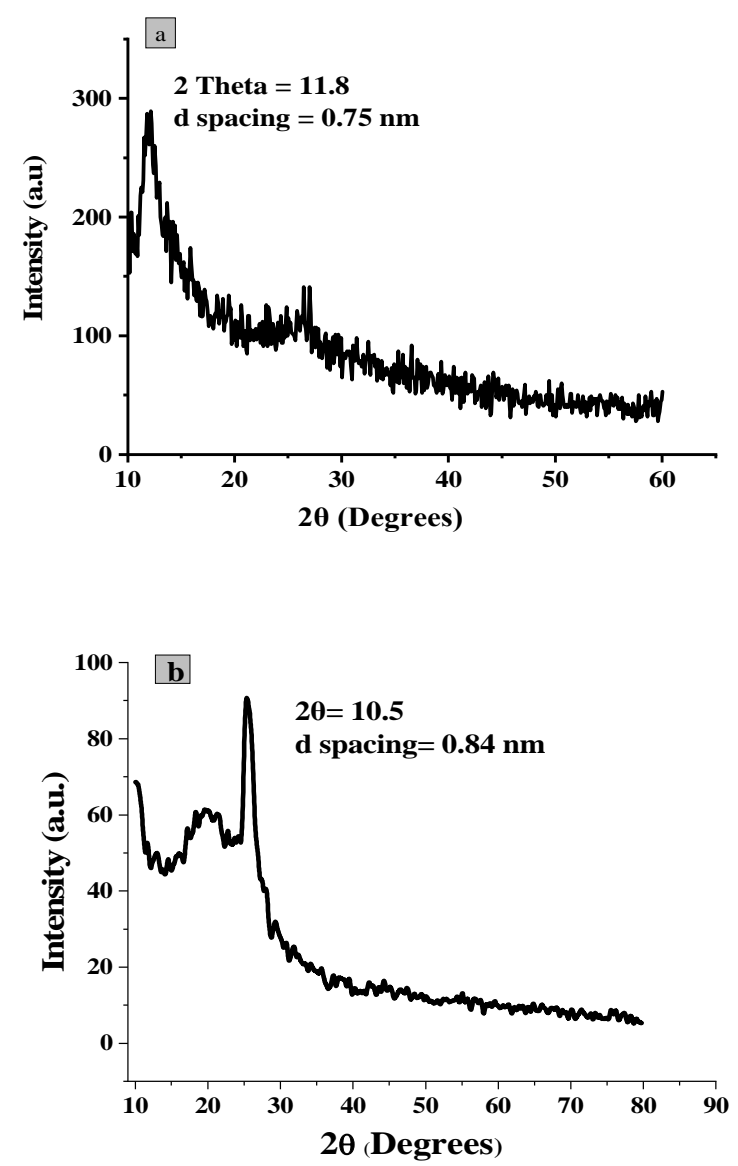

Figure 9. XRD patterns of a) GO, b) S-GO

\section{Gas permeation experiments}

The experimental investigation of flow of $\mathrm{O}_{2} / \mathrm{N}_{2}$ through selective membrane was performed in this work by variation of feed gas pressure. The results of gas permeation test were studied and compiled in the (Table 3 ) in aspect of permeability and selectivity of both gases and the effect of feed gas pressure on these factors. Fig. 10(a-b) depicted the effect of pressure on permeability of both gases and the results revealed that a gradual increase in permeability of oxygen and nitrogen was found with the increase in feed gas pressure from 1 to 5 bars and the best results were obtained at 5 bar pressure in terms of selectivity of membrane. This could be explained in terms of dual-sorption model, which elaborates the direct relation between the permeability of gases with the pressure due to the improved interaction between gas molecules and polymer chains [33].

Table 3. Permeability and selectivity data of synthesized material for $\mathrm{O}_{2} / \mathrm{N}_{2}$ separation.

\begin{tabular}{cccc}
\hline $\begin{array}{c}\text { Pressure } \\
(\mathbf{B a r})\end{array}$ & $\begin{array}{c}\text { Permeability } \\
\text { of } \mathbf{O}_{2} \text { (Barrer) }\end{array}$ & $\begin{array}{c}\text { Permeability } \\
\text { of } \mathbf{N}_{\mathbf{2}} \text { (Barrer) }\end{array}$ & $\begin{array}{c}\text { Selectivity } \\
\left(\mathbf{O}_{2} / \mathbf{N}_{2}\right)\end{array}$ \\
\hline 1 & 1605 & 1573 & 1.020 \\
2 & 2542 & 2325 & 1.093 \\
3 & 3478 & 3125 & 1.113 \\
4 & 3233 & 2851 & 1.134 \\
5 & 4097 & 3373 & 1.215 \\
\hline
\end{tabular}

Generally, an inverse relation is observed in selectivity and permeability of gases through a membrane, higher permeabilities normally led to compromised selectivities and vice versa. However, according to Robinson, the polymeric membranes that can show a trade-off between these two factors are considered to be the best for the purpose [3]. Fig. 10c-d shows the effect of pressure and permeability of gases on their separation factor. An increasing trend is observed in selectivity with increasing the pressure and permeabilities. This could be explained on the basis of the fact that the contribution of TMOPMA groups through solution-diffusion are also facilitating the transport of oxygen gas molecules and thus increasing the selectivity with the permeability. 

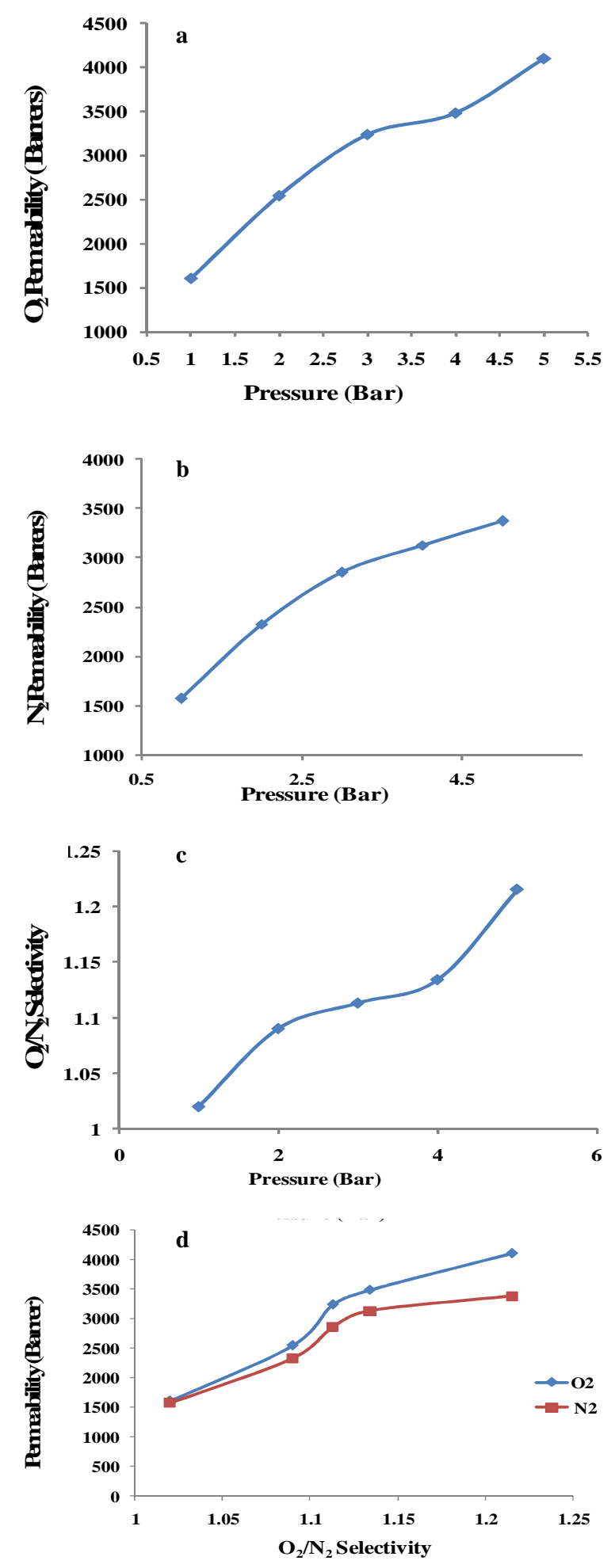

Figure 10. (a) Permeability of $\mathrm{O}_{2}$ (b) Permeability of $\mathrm{N}_{2}$ (c) Selectivity of synthesized MMM for $\mathrm{O}_{2} / \mathrm{N}_{2}$ at different pressures and (d) Relation between selectivity factors of membrane with permeability of both gases

\section{Conclusion}

This study reports the synthesis and applicability of PVC/pAMPS membrane fabricated with TMOPMA modified $\mathrm{GO}$ as inorganic filler for the separation of $\mathrm{O}_{2} / \mathrm{N}_{2}$. The selection of materials for base membrane was made on the basis of their good mechanical strength with superior chemical and physical compatibility with the inorganic filler. The selection of GO for filler was done due to wider applications of it in membrane separations and it was modified with TMOPMA by assuming the contribution of Si-O- groups in the diffusion of oxygen molecules. The synthesized membrane was characterized through different techniques for their morphology and functional group characteristics. The results confirmed the formation of homogeneously dense membranes with all the characteristic functionalities in it. Moreover, the membrane was tested for gas permeation experiments for the separation of $\mathrm{O}_{2}$ and $\mathrm{N}_{2}$ at different pressures. The results revealed the increase in permeability and selectivity with the increase in pressure. Several studies report the selectivity higher than the achieved value, but with very small gas permeabilities. The compromised permeabilities could greatly increase the operation costs. Thus it can be concluded that the synthesized membrane is efficient for its contribution in the separation of $\mathrm{O}_{2} / \mathrm{N}_{2}$ for several industrial applications as it couples the good separation factor with great permeabilities.

\section{References}

1. Y. Kansha, A. Kishimoto, T. Nakagawa and A. Tsutsumi, Sep. Purif. Technol., 77 (2011) 389.

https://doi.org/10.1016/j.seppur.2011.01.012

2. N. Du, H. B. Park, M. M. Dal-Cin and M. D. Guiver, Energy Environ. Sci., 5 (2012) 7306. https://doi.org/10.1039/C1EE02668B

3. L. M. Robeson, J. Memb. Sci., 230 (2008) 390. https://doi.org/10.1016/j.memsci.2008.04.03 $\underline{0}$

4. L. G. Toy, K. Nagai, B. D. Freeman, I. Pinnau and Z. He, T. Masuda, M. Teraguchi 
and Y. P. Yampolskii, Macromolecules, 33 (2000) 2516.

https://doi.org/10.1021/ma991566e

5. N. F. Himma and I G. Wenten. AIP Conference Proceedings, (2017).

090005-1 - 090005-10

doi:10.1063/1.4982313.

6. X. Yang, X. Wang, J. Yang, J. Li and L. Wan, Chem. Phys. Lett., 570 (2013) 125. https://doi.org/10.1016/j.cplett.2013.03.069

7. H. Kim, A. A. Abdala and C. W. Macosko, Macromolecules, 43 (2010) 6515. https://doi.org/10.1021/ma100572e

8. R. K. Joshi, P. Carbone, F. C. Wang, V. G. Kravets and Y. Su, I. V. Grigorieva, H. A. Wu, A. K. Geim and R. R. Nair, Science, 343 (2014) 752. doi:0.1126/science.1245711

9. H. Li, Z. Song, X. Zhang, Y. Huang, S. Li, Y. Mao, H. J. Ploehn, Y. Bao and M. Yu. Science, 343 (2013) 95.

doi:10.1126/science. 1236686

10. S. Hou, S. Su, M. L. Kasner, P. Shah, K. Patel and C. J. Madarang. Chem. Phys. Lett., 501 (2010) 68.

https://doi.org/10.1016/j.cplett.2010.10.051

11. C. Chi, X. Wang, Y. Peng, Y. Qian, Z. Hu,

J. Dong and D. Zhao, Chem. Mater., 28 (2016) 2921.

https://doi.org/10.1021/acs.chemmater.5b04 $\underline{475}$

12. Z. P. Smith and B. D. Freeman. Angewandte Chem. Int. Edition, 53 (2014) 10286.

doi:10.1002/anie.201404407

13. Huang, Q. Liu, N. Wang, Y. Zhu and J. Caro, J. Am. Chem. Soc., 136 (2014) 14686. https://doi.org/10.1021/ja5083602

14. Y. Hu, J. Wei, Y. Liang, H. Zhang, X. Zhang, W. Shen and H. Wang, Angewandte Chem. Int. Edition, 55 (2016) 2048.

https://doi.org/10.1002/anie.201509213

15. S. Castarlenas, C. Téllez and J. Coronas. $J$. Memb. Sci., 526 (2017) 205.

https://doi.org/10.1016/j.memsci.2016.12.04 $\underline{1}$

16. Z. Zhao, H. Xie, S. An and Y. Jiang. J. Phy. Chem. B, 118 (2014) 14640. https://doi.org/10.1021/jp507682k

17. C.-C. Hu, T.-C. Liu, K.-R. Lee, R.-C. Ruaan and J.-Y. Lai, Desalination, 193 (2006) 14. https://doi.org/10.1016/j.desal.2005.04.137
18. W. S. Hummers and R. E. Offeman, J. Am. Chem. Soc., 80 (1958) 1339.

https://doi.org/10.1021/ja01539a017

19. O. Stoilova, N. Koseva, N. Manolova and I. Rashkov, Polym. Bull., 43 (1999) 67. https://doi.org/10.1007/s002890050534

20. N. F. Himma, A. K. Wardani, N. Prasetya, P. T. P. Aryanti and I G. Wenten, Rev. Chem. Eng., 35 (2018) 591.

https://doi.org/10.1515/revce-2017-0094.

21. S. Hafeez, X. Fan, A. Hussain and C. F. Martín, J. Environ. Sci., 35 (2015) 163.

https://doi.org/10.1016/j.jes.2015.04.019

22. M. M. Vuksanović and R. J. Heinemann. Compatibilization of Polymer Blends. (2020) 299.

https://doi.org/10.1016/C2017-0-03891-0

23. Yimin Qin. Medical Textile Materials. (2016) 55.

https://doi.org/10.1016/C2014-0-04473-5

24. A. R. Moghadassi, Z. Rajabi, S. M. Hosseini and M. Mohammadi, J. Ind. Eng. Chem., 20 (2014) 1050. doi:10.1016/j.jiec.2013.06.042.

25. R. S-Delgado1, A.M. S-Delgado1, E. GHernández1, A. O-Paredes1, Z. -Galarza1 and V. M. Castaño. Int. J. Eng. Res. Appl., 6 (2016) 04.

https://www.ingentaconnect.com/content/do aj/22489622/2016/00000006/00000002/art0 $\underline{0007}$

26. K. N. Kudin, B. Ozbas, H. C. Schniepp, R. K. Prud'homme, I. A. Aksay and R. Car, Nano Lett., 8 (2008) 36. https://doi.org/10.1021/n1071822y

27. F. Tuinstra and J. L. Koenig, Raman Spectrum of Graphite, 53 (1970) 1126.

https://aip.scitation.org/doi/abs/10.1063/1.16 74108

28. Z. Zhang, H. C. Schniepp and D. H. Adamson, Carbon, 154 (2019) 510.

https://doi.org/10.1016/j.carbon.2019.07.103

29. R. Brill, Solid State Phy., 20 (1968) 1. https://doi.org/10.1016/S00811947(08)60216-7

30. N. Sharma, V. Sharma, Y. Jain, M. Kumari, R. Gupta, S. K. Sharma and K. Sachdev, Macromol. Symp., 376 (2017) 1700006-1 1700006-5 doi:10.1002/masy.201700006. 
31. Y. Qian, X. Zhang, C. Liu, C. Zhou and A. 33. W. J. Koros, D. R. Paul and A. A. Rocha, J. Huang, Desalination, 460 (2019) 56.

https://doi.org/10.1016/j.desal.2019.03.009

32. I. Castilla-Cortázar, A. Vidaurre, B. Marí and A. J. Campillo-Fernández, Polymers, 11 (2019) 1099.

doi:10.3390/polym11071099

Polym. Sci. Polym. Phys. Edition, 14 (1976) 687.

doi:10.1002/pol.1976.180140410. 